\title{
A SIMPLE ARTIFICIAL NEURAL NETWORK FOR FIRE DETECTION USING LANDSAT-8 DATA
}

\author{
Zhuoran $\mathrm{Liu}^{1}$, Kai Wu ${ }^{1}$, Rui Jiang ${ }^{1}$, Haopeng Zhang ${ }^{1,2,3 *}$ \\ ${ }^{1}$ Department of Aerospace Information Engineering, School of Astronautics, \\ Beihang University, 102206 Beijing, China - zhanghaopeng@ buaa.edu.cn \\ ${ }^{2}$ Beijing Key Laboratory of Digital Media, 102206 Beijing, China \\ ${ }^{3}$ Key Laboratory of Spacecraft Design Optimization and Dynamic Simulation Technologies, \\ Ministry of Education, 102206 Beijing, China
}

\section{Commission III, WG III/4, ICWG III/IVa}

KEY WORDS: Fire Detection, Artificial Neural Network, Fixed Threshold, Landsat-8, Remote Sensing Image Classification

\begin{abstract}
:
Fixed threshold models have been widely used in active fire detection products. However, its accuracy is limited due to the complexity of setting up thresholds. Artificial neural network (ANN) is capable of learning from data and can decide weights automatically. Given enough data, an ANN model is able to optimize itself and quickly find an optimal solution. In this work, a simple ANN model is implemented to classify fire pixels from Landsat- 8 data. Experimental results show that our ANN model effectively achieves fire detection and performs better than fixed threshold model in certain circumstances.
\end{abstract}

\section{INTRODUCTION}

Earth's forest and vegetation suffer greatly due to wildfire or fire caused by human. For example, Australian officials have announced that high temperature and drought have been the main causes of the bush fires in Australia since Australia entered the forest fire season in July 2019. From the most developed and densely populated coastal areas to Western Australia and the northern region, almost every state has been affected by forest fires. The fires have been burning more than 6 months, causing a large number of property losses and casualties of people and animals. Monitoring the fire by remote sensing satellites can detect fires in the early stages, and provide an accurate assessment of direct and indirect fire impacts during and after the fire.

Landsat-8's remote sensing data is one of the main satellite data sources widely used for fire detection, as well as various remote sensing products, including land use detection, vegetation detection, cloud detection, water detection, etc. Compared to other fire products like MODIS (Giglio et al., 2003, Giglio et al., 2018) or VIIRS (Schroeder et al., 2014), Landsat8 equipped with TIRS (thermal infrared sensor) and OLI (operational land imager) imaging instruments has better spatial resolution and lower temporal resolution. Since OLI/TIRS has multiple infrared bands, it is especially useful in thermal detection (Anejionu et al., 2014). In fire detection, a common approach is to analyze the characteristics of the flame and set certain thresholds for the data in each band to filter out pixels with fire. For example, Giglio et al. (Giglio et al., 2003) used midinfrared and long-wave infrared bands to set different thresholds for different responses of scenes containing hot sub-pixel targets to find fire pixels. Fu et al. (Fu et al., 2008) determined the dynamic threshold of fire point by comparing the information of background pixels and fire points. The application of machine learning can also be found in related works (Gautam et al., 2008).

\footnotetext{
* Corresponding author
}

Although fixed threshold model has been widely used in active fire detection products, its accuracy is limited due to the complexity of setting up thresholds. Given enough data, artificial neural network (ANN) can learn from data, decide weights automatically, optimize itself and quickly find an near-optimal solution. In this paper, we propose a simple ANN model to classify fire pixels from Landsat- 8 data. We performed comprehensive experiments to validate the performance of the model and made a comparison with a fixed threshold model.

The rest of this paper is organized as follows. Section 2 shows the study area for Landsat- 8 remote sensing data with fires. Section 3 describes the details of our proposed ANN model. Experimental results are given in Section 4. Finally, Section 5 concludes the paper.

\section{STUDY AREAS}

\begin{tabular}{c|l|c}
\hline Band No. & Wavelength/ $\mu \mathrm{m}$ & Resolution \\
\hline 1 & $0.433-0.453$ & $30 \mathrm{~m}$ \\
\hline 2 & $0.450-0.515$ & $30 \mathrm{~m}$ \\
\hline 3 & $0.525-0.600$ & $30 \mathrm{~m}$ \\
\hline 4 & $0.630-0.680$ & $30 \mathrm{~m}$ \\
\hline 5 & $0.845-0.885$ & $30 \mathrm{~m}$ \\
\hline 6 & $1.560-1.660$ & $30 \mathrm{~m}$ \\
\hline 7 & $2.100-2.300$ & $30 \mathrm{~m}$ \\
\hline 8 & $0.500-0.680$ & $15 \mathrm{~m}$ \\
\hline 9 & $1.360-1.390$ & $30 \mathrm{~m}$ \\
\hline 10 & $10.6-11.2$ & $100 \mathrm{~m}$ \\
\hline 11 & $11.5-12.5$ & $100 \mathrm{~m}$ \\
\hline
\end{tabular}

Table 1. Configuration of OLI/TIRS bands aboard Landsat-8.

Landsat- 8 is a solar synchronous orbit satellite launched by NASA (National Aeronautics and Space Administration) in 2013. The equipped imaging instruments, thermal infrared sensor (TIRS) and operational land imager (OLI), can take 11 bands of images in total, as shown in Table 1. The bands 1 to 7 with $30 \mathrm{~m}$ spatial resolution are usually used for fire identification. 


\begin{tabular}{c|c|c|c|c|c}
\hline Scene No. & Local Time(Y/M/D H:m) & Location & Center Latitude/Longitude & Valid Pixels & Fire Pixels \\
\hline 0 & $2013 / 08 / 2410: 40$ & North America & $37^{\circ} 28^{\prime} 28^{\prime \prime} N / 120^{\circ} 35^{\prime} 08^{\prime \prime} W$ & 634825 & 685 \\
\hline 1 & $2014 / 09 / 1910: 38$ & North America & $38^{\circ} 54^{\prime} 16^{\prime \prime} N / 120^{\circ} 06^{\prime} 54^{\prime \prime} W$ & 634429 & 1146 \\
\hline 2 & $2014 / 07 / 2211: 17$ & North America & $62^{\circ} 51^{\prime} 17^{\prime \prime} N / 113^{\circ} 26^{\prime} 04^{\prime \prime} W$ & 637591 & 5720 \\
\hline 3 & $2014 / 07 / 2911: 14$ & North America & $61^{\circ} 28^{\prime} 39^{\prime \prime} N / 116^{\circ} 01^{\prime} 55^{\prime \prime} W$ & 637334 & 3906 \\
\hline 4 & $2019 / 01 / 3009: 42$ & Australia & $41^{\circ} 45^{\prime} 35^{\prime \prime} S / 147^{\circ} 41^{\prime} 47^{\prime \prime} E$ & 641576 & 449 \\
\hline
\end{tabular}

Table 2. Statistics of scenes in the study area.
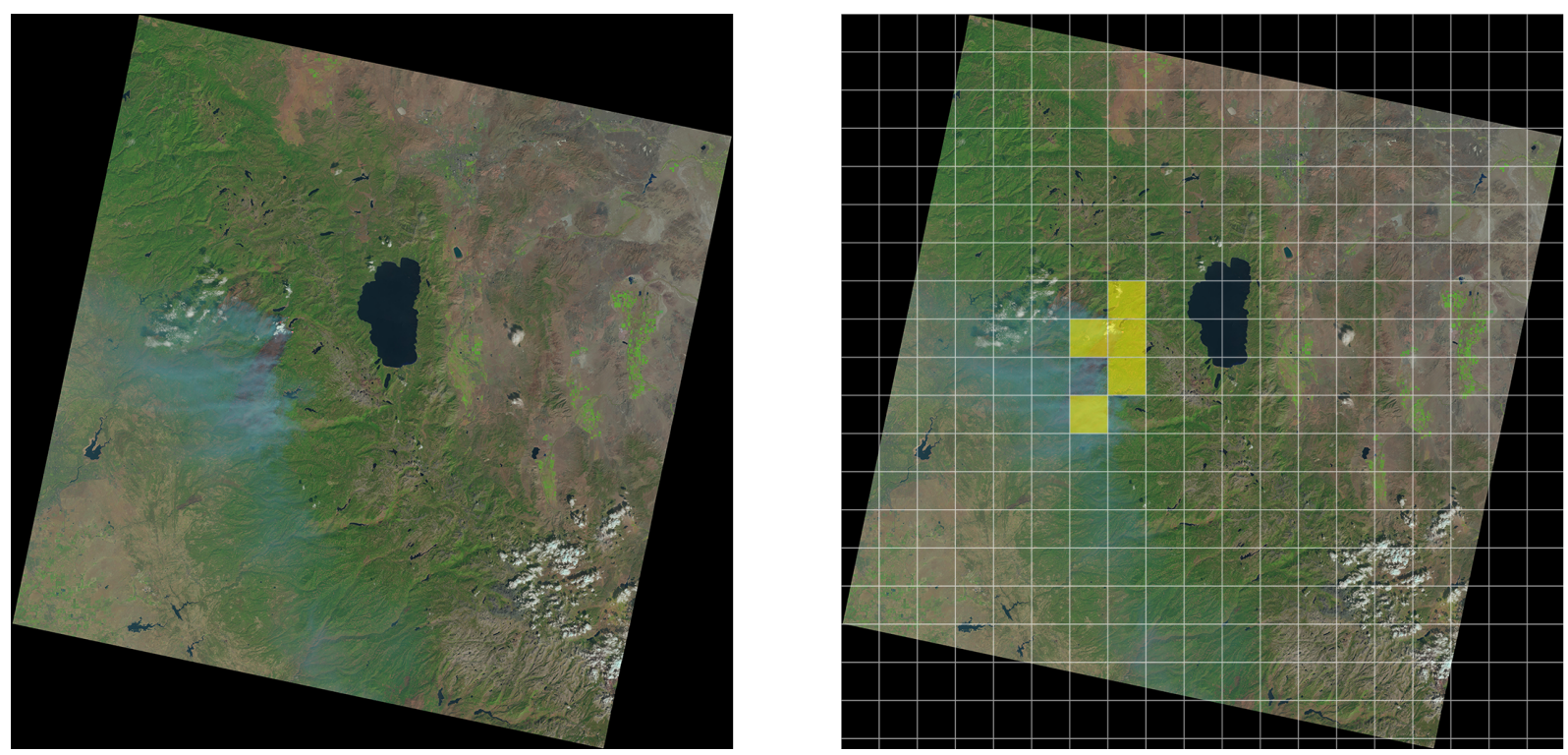

Figure 1. An example of a scene. Left: true color image of scene 1; Right: Scene 1 with 400-by-400 pixels grid. Square images with enough fire pixels inside are filled with yellow color.

The sample images studied in this paper is downloaded via GloVis (Global visualization viewer) from UCGS (United States Geological Survey). The data downloaded include 11 single channel images and one composite visible color image, all of which have a resolution of 30 meters. All the channels are aligned and corrected to UTM (Universal Transform Mercator) projection. The time of acquisition of the five scenes selected are between 9:00 and 12:00 local time. According to the visible image, we manually marked the pixels that can be confirmed as fire, and the other pixels are regarded as the pixels without fire. The statistics of the five scenes are as follows in Table 2.

An example of a scene is given in Figure 1, and an example of marks is shown in Figure 2. Considering the fact that most of valid pixels are non-fire pixels and only a few of pixels are fire pixels, the amount of non-fire pixels should be reduced for balancing the samples in the dataset for training ANN. Thus, when generating negative samples for the training set, only a certain amount of pixels in green areas are selected. When generating negative samples for validation set and test set, scenes are divided into 400-by-400 pieces as shown in the right side of Figure 1. The pieces which have no obvious fire points inside are ignored and only the pieces that contain enough obvious fire pixels are preserved. It should be noticed that we only use four bands of data for fire detection, i.e. band1, band5, band6 and band7. Figure 3 displays images of these four useful bands of one piece.

\section{METHOD}

We implement a simple ANN model to recognize fire points more accurately in Landsat- 8 data. ANN has been widely used

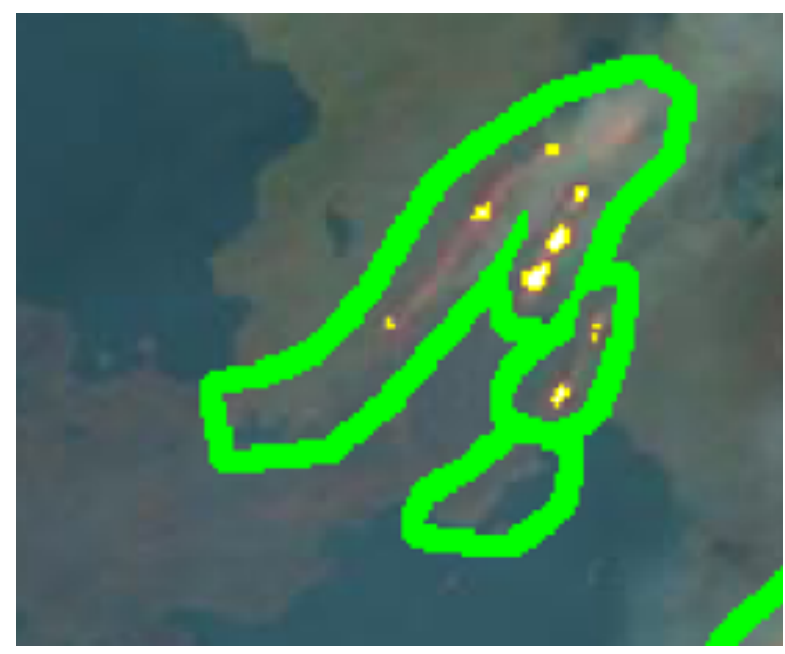

Figure 2. An example of marks. Yellow and white pixels are fire pixels and green pixels are non-fire pixels nearby.

in object detection and has been used in smoke detection and analysis. ANN belongs to artificial intelligence techniques. An ANN consists of many adaptive simple units that are connected together, which is an imitation of biological nervous system's activities. It has great potential to learn different patterns. We expect the model to learn and remember the pixel features of fire points.

When detecting fire points, the multi-spectral data of a single pixel contains adequate information. So it is enough for a full connected neural network (FNN) to learn the features of the fire point. FNN is the basic ANN model which consists of an input 


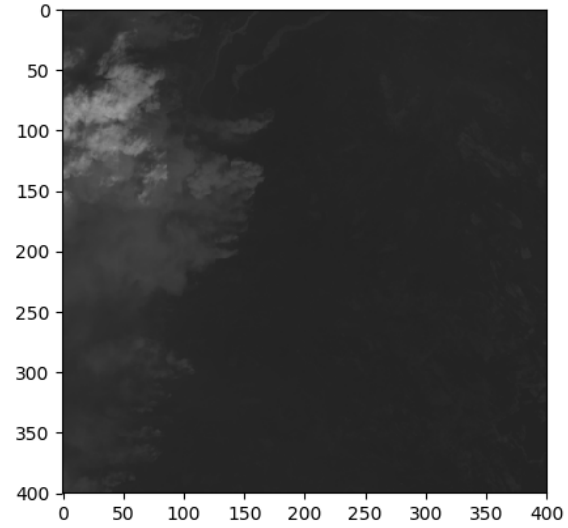

a.

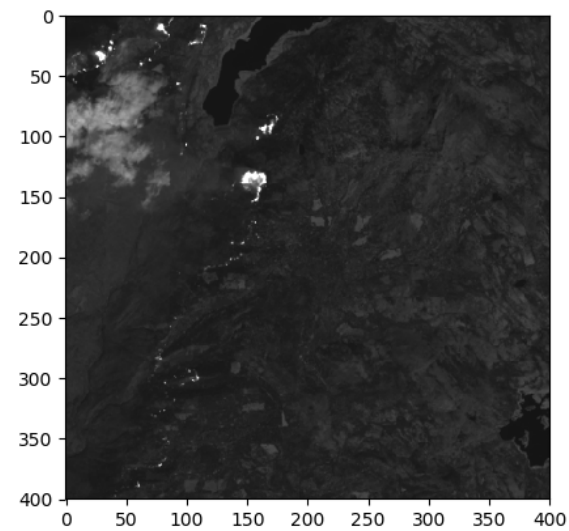

c.

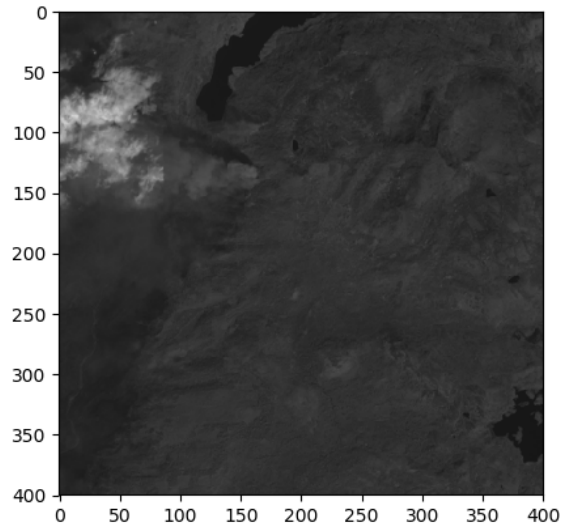

b.

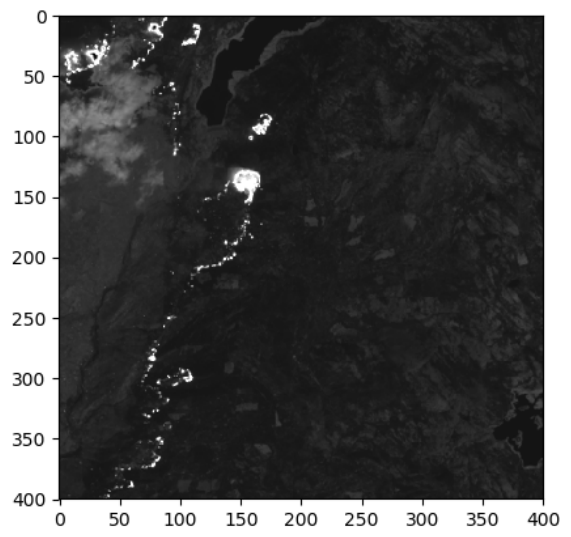

d.

Figure 3. The bands used for fire detection. From a to d: band 1, band 5, band 6, and band 7 .

layer, several hidden layers and an output layer. It is capable of learning features of pixels following simple rules to update the weight in its nodes.

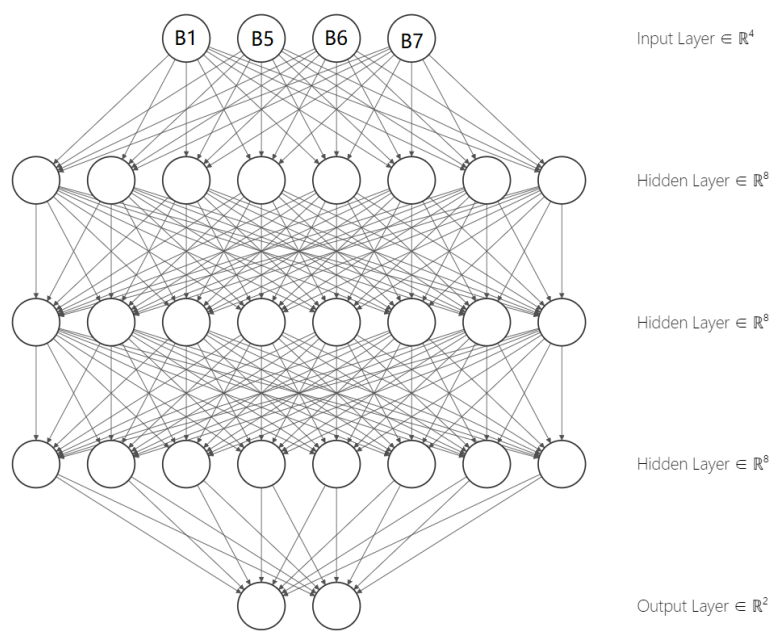

Figure 4. The structure of our ANN model.

As shown in Figure 4, our model consists of an input layer, an output layer and three hidden layers. The input layer has four dimensions, respectively fed with the data of band 1, 5, 6 and 7 from a single pixel. These four bands are selected because they have stronger correlation with fire detection applications
(Schroeder et al., 2016). The output layer uses softmax as activation function while the rest of the network uses tanh as activation function.

Activation functions are very important for artificial neural network models to learn and understand very complex and nonlinear functions. The process of forward propagation can be described as follows.

$$
a_{j}^{i+1}=f\left(\sum_{k=0}^{n_{i}} w_{k, j}^{i} a_{k}^{i}+b^{i}\right)
$$

where $a_{j}^{i}$ is activity of neurons in layer $i, n_{i}$ is the number of neurons in layer $i, w_{k, j}^{i}$ is the weight of layer $i, b^{i}$ is the bias value of layer $i$, and $f$ is the activation function.

\section{EXPERIMENTS}

\subsection{Splitting of Dataset and Criteria of Assessment}

\begin{tabular}{c|c|c}
\hline Scene No. & Positive Samples & Negative Samples \\
\hline 0 & 171 & 9687 \\
\hline 2 & 2235 & 37535 \\
\hline
\end{tabular}

Table 3. Training samples

The statistics of training samples is shown in Table 3. Scene 0 and 2 in our study areas are selected to train the ANN 
model. To avoid confusion between marginal fire pixels and non-fire pixels, an erosion operation is applied to fire pixels and the remaining non-marginal fire pixels are marked as positive samples. Meanwhile, a certain proportion of pixels inside nonfire marked areas are randomly selected to be negative samples.

Scene 1 is selected to validate set. The whole scene is divided into small squares of 400-by-400 pixels as shown in Figure 1. Parameters of the ANN model are optimized according to its performance on the validation set, i.e. scene 1 .

Finally, the testing set is composed of data from scene 3 and 4 , which are also divided into 400 -by-400 squares. Notice that square images with less than 10 fire points are ignored in the reported results.

Performance assessment is based on multiple popular criteria, including precision, recall, overall accuracy $(\mathrm{OA})$ and $F_{1}$ measure, which are defined as follows (Davis, Goadrich, 2006).

$$
\begin{gathered}
\text { precision }=\frac{T P}{T P+F P} \\
\text { recall }=\frac{T P}{T P+F N} \\
O A=\frac{T P+T N}{T P+T N+F P+F N}
\end{gathered}
$$

where $T P, T N, F P$ and $F N$ stand for True Positive, True Negative, False Positive and False Negative, respectively.

$F_{1}$ measure is also a criterion widely used to assess the performance of binary classifiers (Lipton et al., 2014). It can be calculated with precision and recall as

$$
F_{1}=\frac{2 * \text { precision } * \text { recall }}{\text { precision }+ \text { recall }}
$$

Precision stands for the portion of pixels that are real fire pixels among the pixels that a model predicts to be fire pixels. Higher precision means there are fewer commission errors and predicted fire pixels are more likely to be true. Recall stands for the portion of pixels that are predicted to be fire pixels among the pixels that are real fire pixels. Higher precision means there are fewer omission errors and the model is less likely to ignore real fire pixels. In this circumstance, ignoring real fire pixels

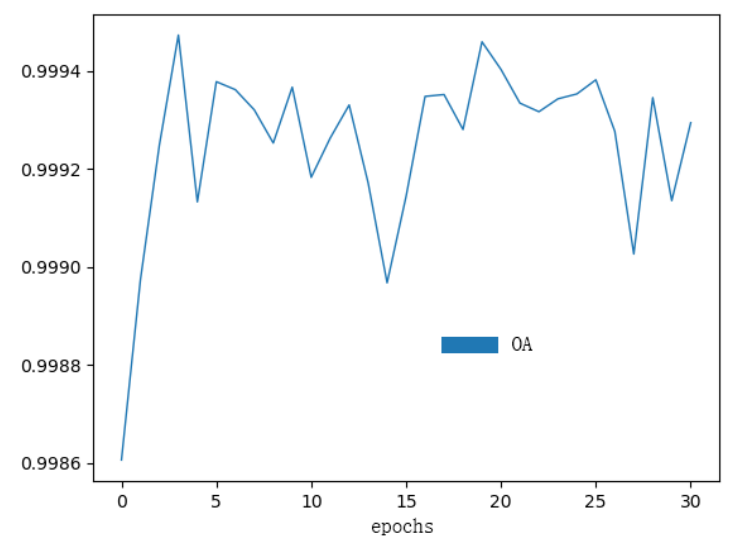

Figure 5. Overall accuracy in training epochs.

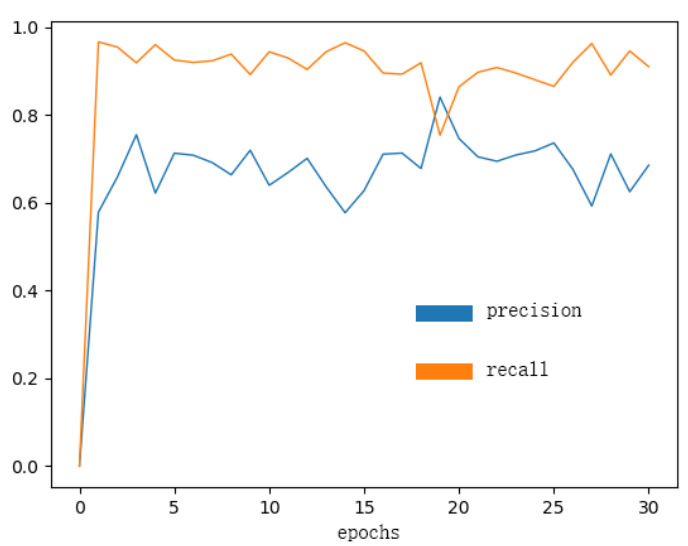

Figure 6. Precision and recall in training epochs.

is more costly than predicting fake fire pixels, so recall is considered more important than precision.

\subsection{Training Result of ANN}

We select the Adam optimizer to train our ANN model with a learning rate of 0.001. Figure 5 and Figure 6 show the performance of our ANN model as training epochs increases. Precision, recall and overall accuracy are calculated using validation set and the confidence threshold is set to $\lambda=0.5$. We can see that precision and recall reach peak values after 1-2 epochs. However, after 3 epochs, both values start to oscillate. When precision increases, recall decreases. This suggests that the training process has reached its limit and more training will not further increase the overall performance of the model. The performance on the training set also indicates that the model tends to converge after 30 epochs.

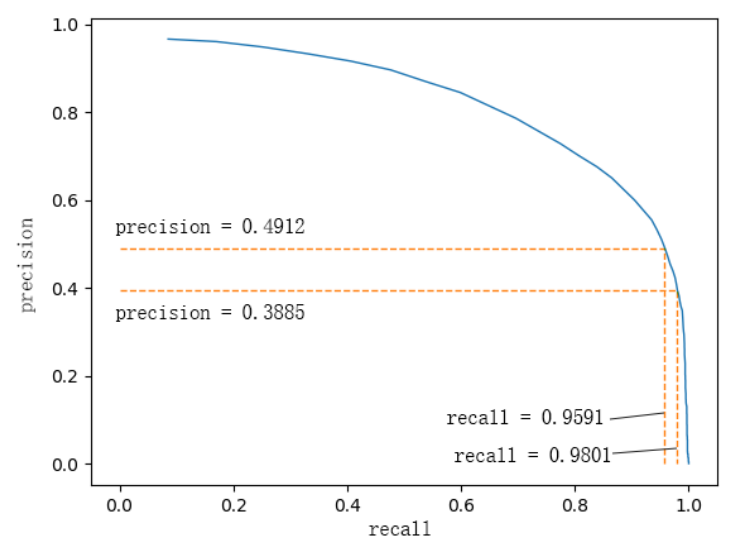

Figure 7. Precision-Recall curve of the ANN model's outputs

With different confidence thresholds, the ANN model presents different precision and recall values, which are shown by the P$\mathrm{R}$ curve in Figure 7. Every point on the P-R curve represents a possible solution for the classification task. The area under the P-R curve represents the average precision of when different confidence thresholds are selected (Sahiner et al., 2017). In this case, our average precision is 0.8267 . 


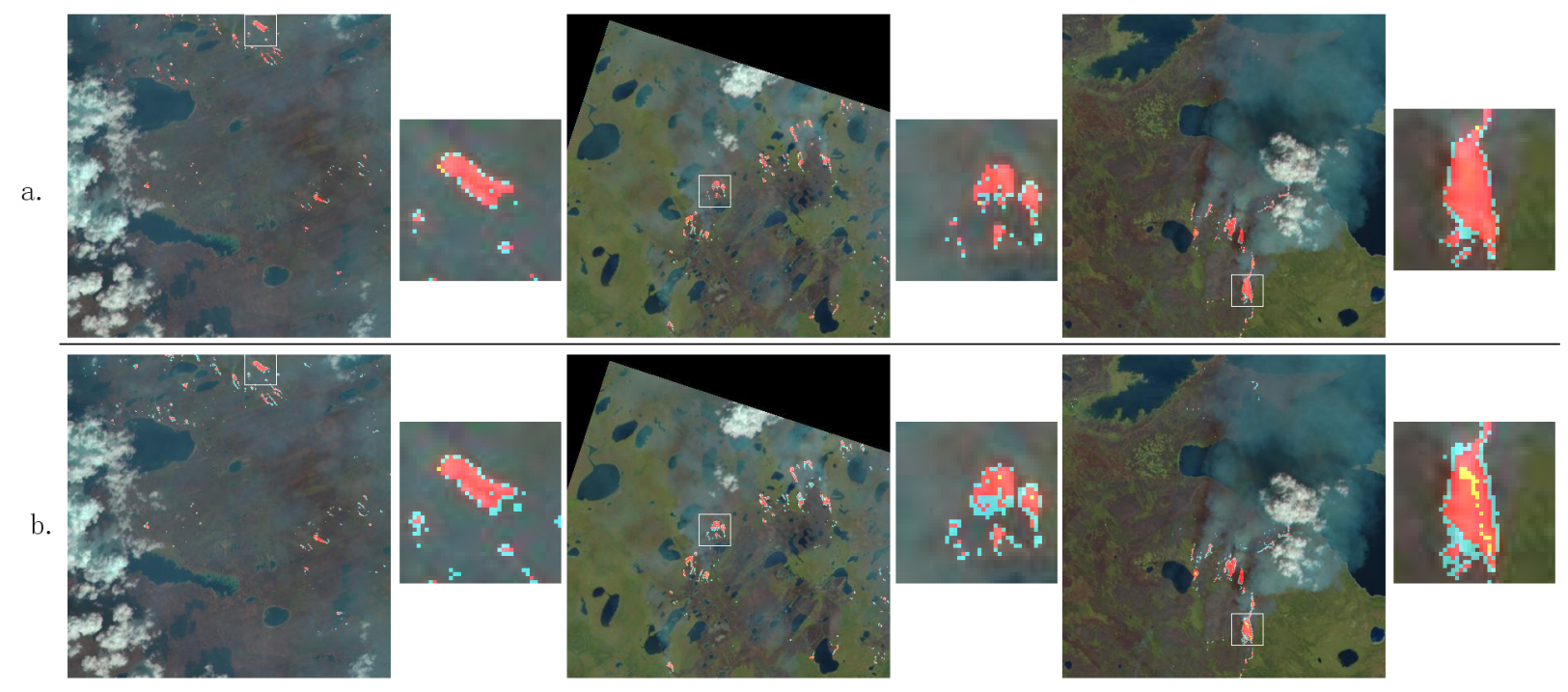

Figure 8. The visualized comparison of two models. Subfigure a. shows the prediction of our ANN model and subfigure b. shows the results of the fixed threshold model. Red pixels stand for true positive, yellow pixels stand for false negative, cyan pixels stand for false positive, and the rest of pixels are true negative.

\subsection{Comparison}

A fixed threshold model (Schroeder et al., 2016) is adopted as a reference to evaluate the performance of our ANN model. The fixed threshold model (Schroeder et al., 2016) also uses brightness data from bands 1, 5, 6 and 7 as its input, as well as additional the mean value and standard deviation of the nearby area. Certain thresholds are tuned to achieve the best performance of the fixed threshold model on the validation set. The model is then tested on the testing set. The results are shown in Table 4 .

\begin{tabular}{c|l|l|l|}
\multicolumn{1}{c}{} & \multicolumn{2}{c}{ Truth } \\
\cline { 2 - 4 } \multicolumn{1}{c}{} & fire & non-fire \\
\cline { 2 - 4 } Prediction & fire & 3802 & 5985 \\
\cline { 2 - 4 } & non-fire & 162 & 3386701 \\
\cline { 2 - 4 } & &
\end{tabular}

Table 4. Testing results of the fixed threshold model.

To better demonstrate the difference between the fixed threshold model and our ANN model, several typical square images of 400-by-400 pixels are selected and colored according to the results of the two models, as illustrated in Figure 8. The two models output similar results in most regions. The number of false positive pixels is relatively large and the number of false negative pixels is relatively small, which is a good feature for fire detection models. However, in specific areas, there are still slight differences. The fixed threshold model tends to mistakenly regard more non-fire pixels around fire pixels as fire pixels. Also, the fixed threshold model may tend to have omission errors at the center of large fires. This is possibly caused by over saturation (Morfitt et al., 2015) caused by excessive amounts of radiation. Because the center of fire generates too much radiation, the signal that the sensor received goes far beyond the limitation of the sensor, and the sensor returns a very low value of radiance instead of outputting large values. In the fixed threshold model, this creates huge difficulty for debugging since it is hard to define an over saturated pixel. Although output implementation of the fixed threshold model has already included the criteria specially set up to identify over saturated pixels, omission errors still exist. However in ANN model, as long as over saturated pixels are included in training set, the model will try to adapt to the data. From the visualization comparison in Figure 8, ANN shows better ability to handle special situations.

\begin{tabular}{c|c|l|l|l|l}
\hline \multicolumn{2}{l|}{} & Precision & Recall & OA & $F_{1}$ \\
\hline \multicolumn{2}{l|}{ Fixed Threshold } & 0.3885 & 0.9591 & 0.9982 & 0.5530 \\
\hline \multirow{2}{*}{ ANN } & $\lambda=0.6385$ & 0.4912 & 0.9591 & 0.9988 & 0.6497 \\
\cline { 2 - 6 } & $\lambda=0.4345$ & 0.3885 & 0.9801 & 0.9981 & 0.5564 \\
\hline
\end{tabular}

Table 5. Result comparison of two models.

On the testing set, both ANN and fixed threshold models have relatively high recall rate and relatively low precision. As shown in Table 5 and Figure 7, if we choose $\lambda=0.6385$ as the confidence threshold, the ANN model gets a recall of 0.9591 and a precision of 0.4912 . In this situation, the recall of the ANN model equals the recall of the fixed threshold model while the precision of the ANN model appears to be better than that of the fixed threshold model. From another perspective, if we choose $\lambda=0.4345$ as the confidence threshold, the ANN model shows a recall of 0.9801 and a precision of 0.3885 . The precision of the ANN model equals the precision of the fixed threshold model while the recall of the ANN model is higher in comparison to that of the fixed threshold model. To summarize, the ANN model has better overall performance on the testing set.

From the perspective of running time, our implementation of the fixed threshold model takes 6.9 seconds on average to process a image of 400-by-400 pixels while the ANN model takes 3.8 seconds on average to predict the same data. The ANN model is more efficient, probably because the fixed threshold model requires time consuming calculation of the average and standard deviation around each pixel while the ANN model does not.

\section{CONCLUSION}

In this paper, a simple ANN model using multi-spectral data from Landsat- 8 has been proposed to detect fire pixels. According to the performance comparison with the fixed threshold 
model, our ANN model shows better compatibility with details like over saturated pixels at the center of large fires. The feature of the ANN model that it automatically improves itself with data, could lower the chance of human mistakes compared to manually designing fixed thresholds. However, ANN still has uncertainty when training, and fire pixels still need to be marked manually, which is inconvenient. Further study is needed to solve these problems.

\section{ACKNOWLEDGEMENTS}

This work was supported in part by the National Key Research and Development Program of China (Grant Nos. 2016YFB0501300 and 2016YFB0501302), the National Natural Science Foundation of China (Grant No. 61501009), and the Fundamental Research Funds for the Central Universities.

\section{REFERENCES}

Anejionu, O. C. D., Blackburn, G. A., Whyatt, J. D., 2014. Satellite survey of gas flares: Development and application of a Landsat-based technique in the Niger Delta. International Journal of Remote Sensing, 35(5), 1900 - 1925.

Davis, J., Goadrich, M., 2006. The relationship between precision-recall and roc curves. ICML 2006 - Proceedings of the 23rd International Conference on Machine Learning, 2006, Pittsburgh, PA, United states, 233 - 240.

Fu, Y., Su, Y., Zhong, X., 2008. Forest fire fire detection method based on MODIS remote sensing image. Journal of South China Normal University (Natural Science Edition),, 2008(03), 112-118.

Gautam, R., Singh, D., Mittal, A., Sajin, P., 2008. Application of SVM on satellite images to detect hotspots in Jharia coal field region of India. Advances in Space Research, 41(11), 1784 1792.

Giglio, L., Boschetti, L., Roy, D. P., Humber, M. L., Justice, C. O., 2018. The Collection 6 MODIS burned area mapping algorithm and product. Remote Sensing of Environment, 217, $72-85$.

Giglio, L., Descloitres, J., Justice, C. O., Kaufman, Y. J., 2003. An Enhanced Contextual Fire Detection Algorithm for MODIS. Remote Sensing of Environment, 87(2), 273 - 282.

Lipton, Z. C., Elkan, C., Naryanaswamy, B., 2014. Optimal thresholding of classifiers to maximize f1 measure. Lecture Notes in Computer Science, 8725 LNAI, Nancy, France, 225 -239 .

Morfitt, R., Barsi, J., Levy, R., Markham, B., Micijevic, E., Ong, L., Scaramuzza, P., Vanderwerff, K., 2015. Landsat-8 Operational Land Imager (OLI) Radiometric Performance OnOrbit. Remote Sensing, 7(2), 2208-2237.

Sahiner, B., Chen, W., Pezeshk, A., Petrick, N., 2017. Comparison of two classifiers when the data sets are imbalanced: The power of the area under the precision-recall curve as the figure of merit versus the area under the roc curve. Progress in Biomedical Optics and Imaging - Proceedings of SPIE, 10136, Orlando, FL, United states.
Schroeder, W., Oliva, P., Giglio, L., Csiszar, I. A., 2014. The New VIIRS 375m active fire detection data product: Algorithm description and initial assessment. Remote Sensing of Environment, 143, 85 - 96.

Schroeder, W., Oliva, P., Giglio, L., Quayle, B., Lorenz, E., Morelli, F., 2016. Active fire detection using Landsat-8/OLI data. Remote Sensing of Environment, 185, 210 - 220. 